\title{
Trace Element Micro-Analytical Imaging via Laser Ablation Inductively Coupled Plasma Mass Spectrometry (LA-ICP-MS)
}

Angus Netting, Justin Payne, Benjamin Wade and Tom Raimondo

University of Adelaide, SA, Australia

Traditionally LA-ICP-MS has been used to quantify trace element ( $\mathrm{wt} \%$-ppb) concentrations in a wide variety of materials via spot analysis. This involves focussing a high energy pulsed UV NdYAG laser onto the region of interest on the surface of the sample, upon which the material is ablated. The ablated material is then transported into the argon plasma where it is broken down into constituent atoms and then ionised, whereupon it passes into the mass spectrometer and the elements of interest are analysed. In addition to spot analysis, the software allows rastering of the focussed laser beam in transects whereupon intensity and spatial distribution of elements can be recorded. Post acquisition processing then allows the transect data to be compiled into an image of elemental intensity.

Existing instrumentation used to investigate trace element distribution in mineral and biological samples include EPMA, TEM, SEM, Ion Microprobe, Proton Microprobe, and synchrotron XRF analysis. All these techniques have their own benefits, but also their own inherent limitations whether they be high detection limits $(>0.1-1.0 \mathrm{wt} \%)$, very small analytical areas, complex sample preparation, and/or prohibitively expensive instrumentation. Imaging via LA-ICP-MS fits a niche in which its spatial resolution will never compare to electron beam techniques, however it has superior detection limits ( $\mathrm{ppb}$ ) over a wide elemental range (7Li to 238U), and can analyse a wide variety of materials with minimal sample preparation required. This study seeks to compare Laser Ablation and Electron Probe mapping. The different paradigm required for mapping using laser ablation ICPMS is discussed. It also demonstrates LAICPMS mapping of a range of samples, which experimentally are very difficult to analyse using traditional electron beam techniques.

The ability to map trace elements and specific isotopes offers important information to researchers. Figure 1 following demonstrates different zoning characteristics in a garnet from a shear zone found when comparing major (1-3 wt \%) and trace (ppb-ppm) elements found using both EPMA and LAICPMS mapping. These findings provide valuable information on elemental diffusion due to fluid movement in geological shear systems. 


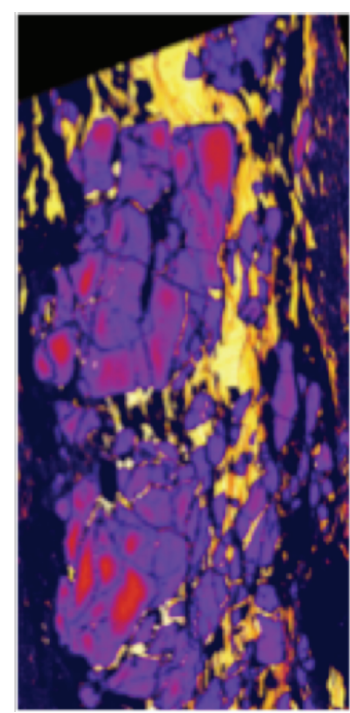

$\mathrm{Mg}$

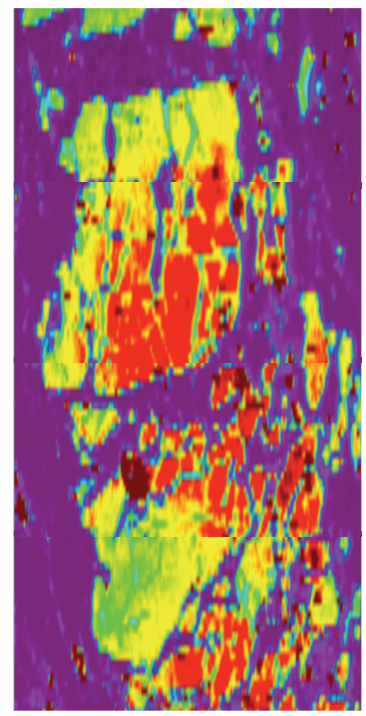

Dy

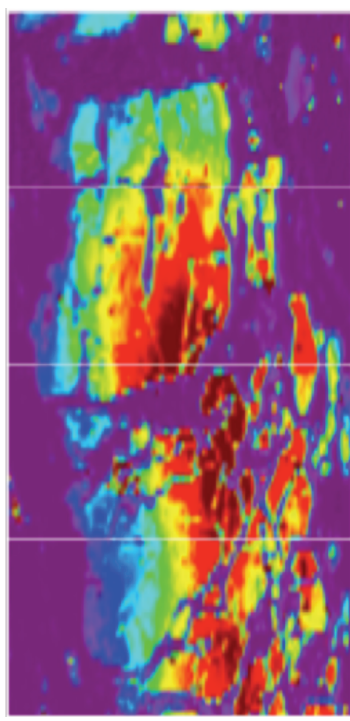

$\mathrm{Yb}$

Figure 1: Elemental distribution of major and trace elements of an almandine garnet comparing Mg Ka (EPMA) with ${ }^{163} \mathrm{Dy}$ and ${ }^{172} \mathrm{Yb}$ (LAICPMS). 